\title{
The Orthopoedic Management of Myelomeningocoele
}

\author{
J. E. HANDELSMAN, M.Ch. Orth., F.R.C.S. \\ Principal Orthopoedic Surgeon, General Hospital, Transvaal Memorial Hospital for Children \\ and University of the Witwatersrand, Johannesburg.
}

In the 4th Century B.C., Hippocrates made the first written reference to spina bifida in Liber Glandulis but it was not until the 16th Century that Vesalius accurately described a boy with multiple deformities of the lower limbs and an associated hydrocephalus.

The pathology of myelomeningocoele, associated hydrocephalus and renal complications is fully dealt with elsewhere in this Symposium, but it is worth emphasising the importance of early surgical closure of the spinal defect in order to preserve existing neurological tissue.

\section{THE SPINAL DEFECT}

The degree of paraplegia seen in the myelomeningocoele patient depends primarily on the level of the lesion, and in general terms, the higher the defect the more profound is the paralysis and sensory deficit in the lower limbs.

At any level, however, cord and nerve roots may be functioning in spite of the spinal lesion and herniation but this function will be lost unless the exposed sac is covered by skin within the first few hours of life. Achieving closure often presents a formidable surgical problem because of deficient soft tissues and the widened bifid posterior spinous processes. Recently a technique has been developed whereby unwanted bone is removed from around the nerve roots, and even whole vertebral bodies excised (Sharrard, 1968). This greatly facilitates skin closure, and is of particular value when a gibbus is present. This deformity occurs in one in eight patients, and correction in the neonatal period prevents the perpetuation of a grotesque deformity that may subsequently require correction by a difficult spinal osteotomy (Fig. 1).

\section{ASSOCIATED DEFORMITIES}

The association of dislocation of the hip, equino-varus, valgus and calcaneus deformities of the foot have long been recognised in myelomeningocoele. Early workers regarded these as idiopathic but unconnected congenital abnormalities, but more critical observation has revealed specific patterns of deformity depending upon the level of the spinal lesion.

The commonest type of myelomeningocoele obliterates all segments from the fourth lumbar neurological level distally. The attitude of such a child at birth is characteristic. Fixed flexion deformity of the hips is constant and dislocation of the hips is frequently present at birth. The knees are held extended and externally rotated. Deformities of the feet vary, but are usually calcaneo- or equino-varus (Fig. 2). On the other hand children with a spinal lesion in the lumbo-dorsal region never present with fixed contractures, dislocations or deformities at birth. Furthermore, low lumbar and sacral lesions affect the feet only. It is thus apparent that deformity is a result of partial innervation of the lower limb rather than total absence, and certainly is not idiopathic in nature. It is reasonable to infer that muscle imbalance is the fundamental cause of the associated deformities. Because this imbalance commences during intra-uterine life, the deformities appear "congenital".

\section{SENSORY CHANGES}

Unlike poliomyelitis, where muscle imbalance may lead to similar deformities, the child with a myelomeningocoele has lost normal skin sensation in the distribution of the nerve roots involved. Because protective pain appreciation is absent, treatment that involves corrective pressure however gentle, is precluded.

An aspect of the sensory deficiency not always appreciated is the loss of proprioception. Because these patients do not know where their lower limbs are in space, rehabilitation is difficult. Sometimes cerebral damage, produced by the hydrocephalus, compounds the lack of adequate joint and muscle sensation. Even with the most active rehabilitative programme, walking may be delayed for some years.

\section{ASSESSMENT}

Estimation of the degree of paralysis and sensory loss at the earliest opportunity enables the practitioner to plan for the future, and judge the effect of immediate closure of the spinal defect.

In a new-born child assessment of muscle power and sensation is extremely difficult. However, deformity is readily apparent, and the range of movement of each joint may be accurately observed and recorded. Both these features give a good indication of the type of muscle imbalance that is already present at birth and it is the practice at the Spinal Defects Clinic of the Transvaal Memorial Hospital for Children to chart these clinical signs at the first visit.

Even in the very young patient a fair assessment of muscle power may be made. A warm and wakeful child will move all normal limbs and simple observation will reveal a muscle group that is not functioning adequately. Skin stimulation may provide an indication of the sensory loss.

With each visit the examination should be repeated and eventually a complete picture of the motor and sensory deficit will emerge. By studying the nerve root innervation of the lower limb muscles, inferences can be drawn where a common spinal level caters for more than one group. For example the medial hamstring muscles may be easily examined, and when paralysed, it may be deduced that the hip abductors, which are not easy to examine will also be paralysed (Sharrard, 1964).

This early assessment provides a good guide to possible future deformity, the potential for walking unaided or the need for assistance with suitable appliances at a later date.

\section{PRINCIPLES OF MANAGEMENT}

In the past a large proportion of myelomeningocoele children have been condemned to a wheel-chair existence. The apparently "congenital" deformities resisted the usual forms of treatment because the underlying cause-muscle imbalance-was neither appreciated nor corrected.

The modern surgeon finds this approach untenable. $\mathrm{He}$ expects to rebalance the lower limbs so that every patient may learn to stand and walk independently.

To achieve this, the patient must have plantigrade feet, extended knees and stable hips, and a balanced upright trunk. Correction of the feet takes precedence because once a plantigrade base is extablished, standing and walking with assistance is possible and rehabilitation may commence. With the feet corrected, appliances may be fitted to overcome weakness of the knees and hips. Although flexion contractures and dislocation of the hips tend to tip the trunk forwards, increasing the lumbar lordosis may compensate this tendency sufficiently to make walking feasible. The importance of achieving the upright posture as early as possible and training the balance mechanisms cannot be over-emphasised. 


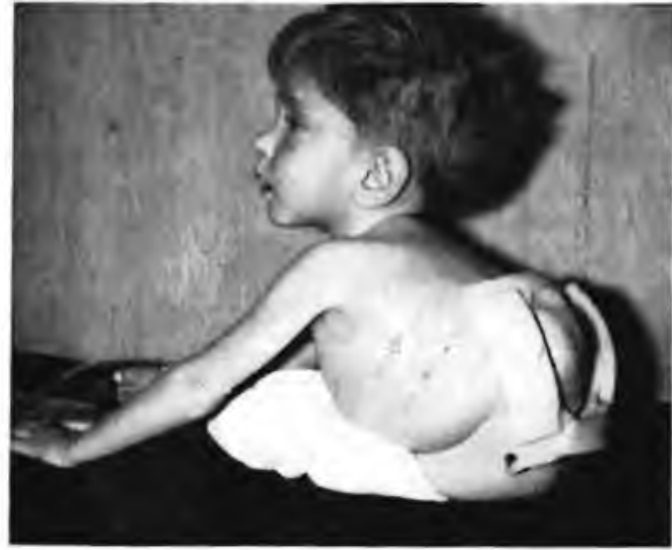

$1 \mathrm{a}$
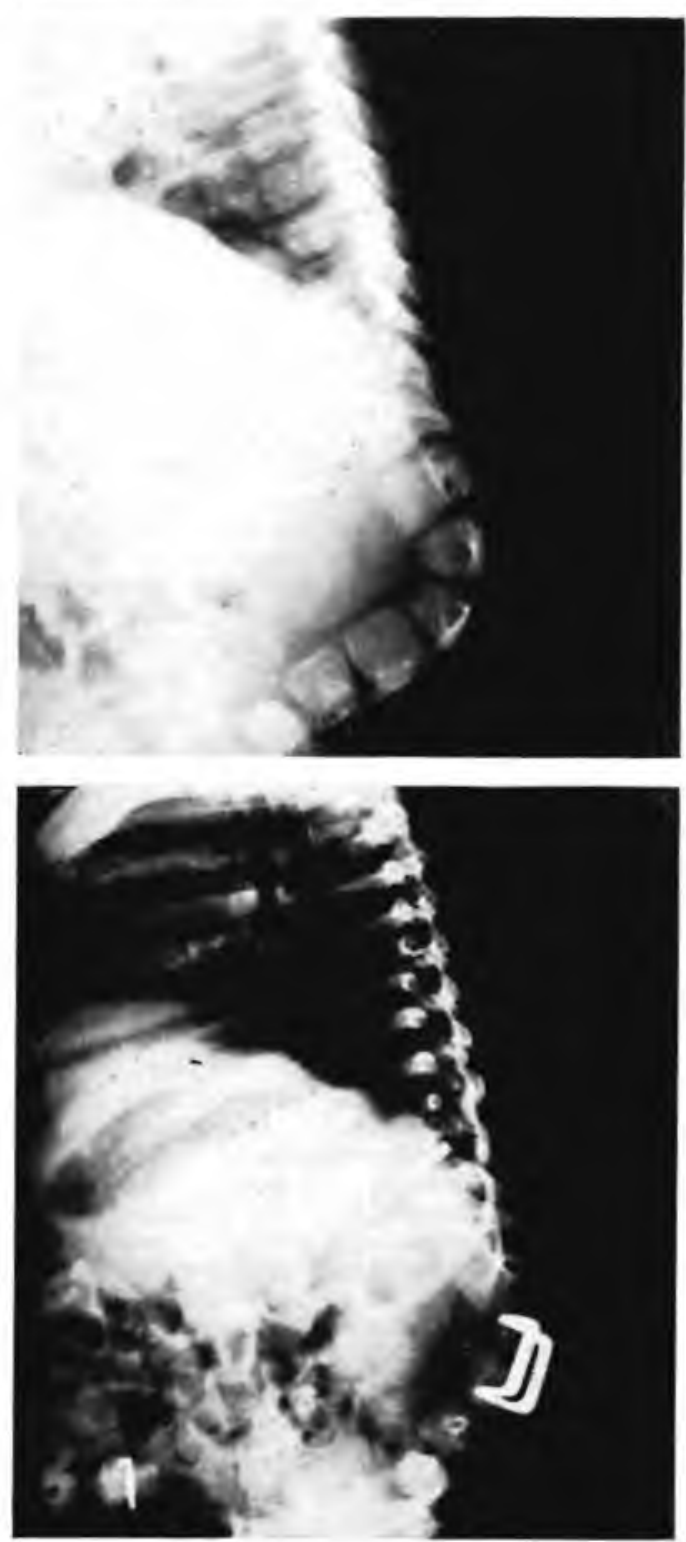

16
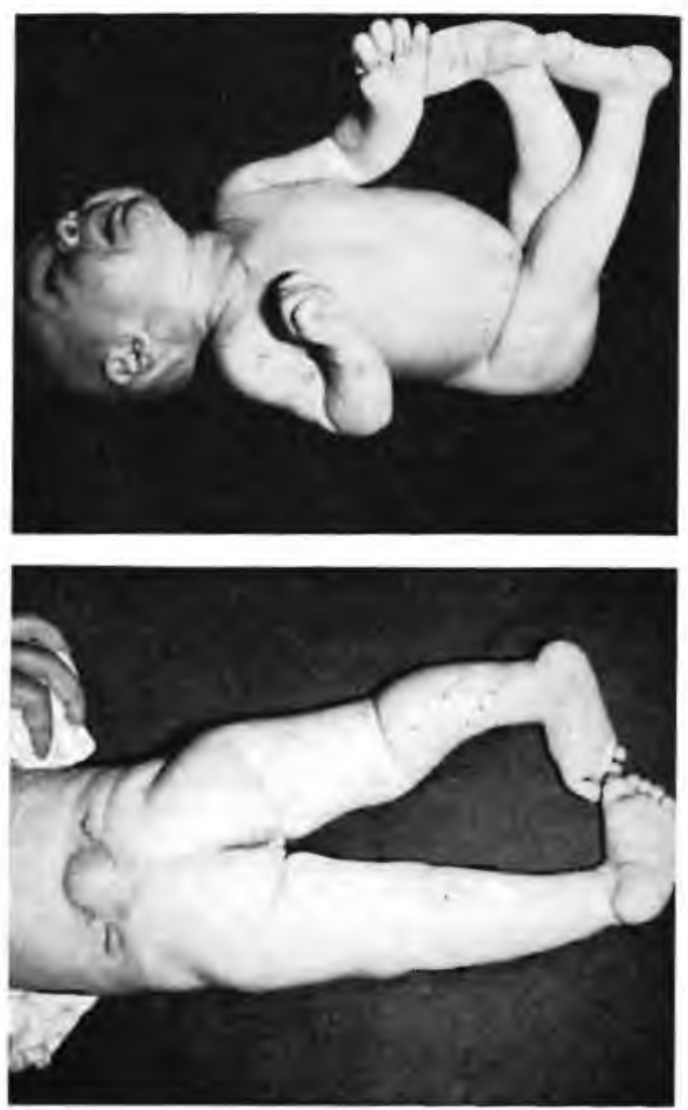

Lack of normal protective skin sensation imposes a different therapeutic approach to the correction of deformity. Even the slightest pressure applied by a padded plaster of Paris cast readily produces pressure necrosis of the skin. The subsequent penetrating ulcers may take months and even years to heal, and if osteitis supervenes amputation may be the sequel. For these reasons the use of calipers or splints as corrective measures are ineffective and dangerous, as are frames and traction. Once correction has been achieved by surgery however, calipers and night splints may be used to maintain correction but must be removed frequently during the course of a day so that early skin erythema is not missed.

Manipulations under anaesthesia are equally unsatisfactory. Even when deformities are corrected by this method, the underlying muscle imbalance will produce a rapid recurrence of the original deformity. Furthermore, the lower limb bones of these patients are abnormal and fracture readily - often spontaneously. For this reason, manipulation is not only ineffective but dangerous.

Thus fixed deformity requires surgical intervention for satisfactory correction. Operations may be commenced as soon as the child's general condition permits. Particularly

Fig. 1: A severe lumbar gibbus in a 5-year old boy ( $a$ and $b$ ) treated by spinal osteotomy with excision of one-and-a-half vertebral bodies and staple fixation (c).

Fig. 2: The typical attitude of an infant with a mid-lumbar myelomeningocoele. The hips are dislocated, fixed in flexion and external rotation, and the knees extended (a). The left foot is held in equino-varus and the right shows a hind-foot equinus deformity (a and $b$ ). 
with regard to the foot, correction should be achieved as early as possible if normal bony anatomy is to be maintained. The simple division of tight soft tissues and lengthening of shortened tendons will not achieve a long-term correction. The original muscle imbalance, however slight, will cause irrevocable relapse. It is essential therefore to rebalance a joint by transferring a relatively over-active tendon that is producing deformity to a situation where it will have the opposite effect. Unlike the muscle imbalance in poliomyelitis, it is acceptable to transfer quite weak muscles in order to remove a deforming force, however slight. Furthermore, in the new position the transferred tendon will often provide a tenodesis effect and does not stretch, because the muscle bellies in the myelomeningocoele are frequently fibrous.

Where there is no deformity but only weakness or paralysis, it is rarely possible to achieve stability by muscle balancing. In these situations, an external appliance such as a caliper is required.

The more complicated hip surgery is frequently delayed until maximum development of spared muscles has been achieved, and rehabilitation is well under way.

\section{THE MANAGEMENT OF SPECIFIC DEFORMITIES} The Feet

Besides providing the patient with plantigrade feet before walking commences, early surgery improves the prognosis. A severe fixed foot deformity at birth represents a dislocation rather than distortion of actual bone structure. With persistence of the deformity, however, alteration in shape of the tarsal bones occurs. These have little capacity for remodelling and because the ultimate prognosis for the foot depends upon maintaining a normal bony configuration, correction as early as possible is imperative. Surgical correction may be commenced at the age of three months or less in a fit child.

The specific foot deformity seen depends upon the particular muscle imbalance present and it is noteworthy that slight residual function in one muscle group in an apparently flail foot is enough to produce a severe fixed contracture. Thus a functioning triceps surae but relatively weak foot extensors will produce an equinus deformity of the calcaneum, whereas the opposite form of muscle imbalance will irrevocably draw the heel into calcaneus. Forefoot adduction and varus usually go together and are largely a function of relative over-action of the tibialis posterior. When the peroneal tendons predominate valgus and even abduction of the forefoot will result. An isolated intrinsic muscle paralysis seen with lesions at the SI level, may produce clawing of the toes at a very early age and cavus of the foot by adolescence.

\section{The Equino-Varus Foot}

The commonest foot deformity in the myelomeningocoele child is equinovarus. This closely resembles the common congenital club foot and has in the past led to the belief that its occurrence was a separate congenital anomaly.

The surgical release follows the principles applied to the common club foot (Solomon and Handelsman, 1967) but a tendon transfer is always included.

The foot is approached from the medial aspect and elongation of the tendo-calcaneus, flexor hallucis longus and flexor digitorum Iongus tendons are carried out. In addition, division of the posterior capsule of the ankle joint and the medial tarsal capsules especially around the navicular completes the procedure. The release alone will not prevent a relapse, so balance is sought. The tibialis posterior tendon is divided at its insertion into the navicular at the time of the release operation and transposed through the interosseous membrane to the dorsum of the foot, providing opposition to the equino-varus tendency (Fig. 3).

\section{The Equino-Valgus Foot}

When the forefoot is relatively flail, but the tendocalcaneus short, the patient's effort to achieve a plantigrade
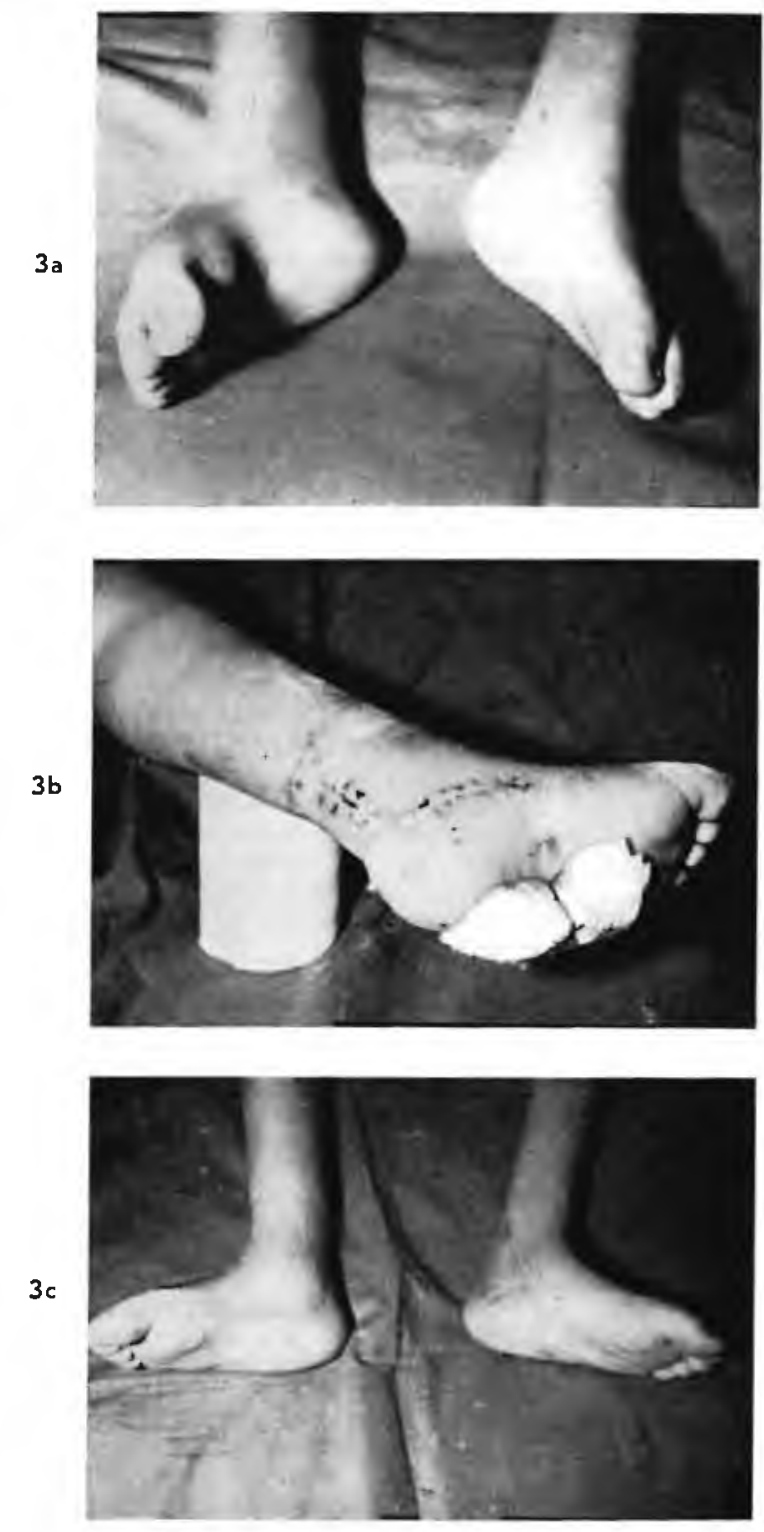

position will force the forefoot into a valgus position. In these cases a simple elongation of the tendo-calcaneus with release of the posterior capsule of the ankle joint may suffice. In most instances, however, the forefoot valgus is the result of relative over-action of the peroneal muscle group in relation to the tibialis posterior. When this imbalance is severe and the equinus marked the foot becomes "broken" around the head of the talus which is drawn into a vertical position by the high calcaneum. The foot then assumes a rocker-bottom shape, the patient walks on the dislocated head of talus rather than the calcaneum and a

Fig. 3: The common equino-varus deformity (a), treated by a medial and posterior capsular release and tendon elongation. The tibialis posterior tendon has been transposed to the lateral side of the foot and is held, in a tunnel made in the tarsal bones, by a silk suture tied over a gauze pad which is left undisturbed for 6 weeks (b). A stable plantigrade foot is the result (c). 
disabling and ugly deformity ensues. The appearances of this foot are virtually identical to the extreme form of congenital flat foot or congenital vertical talus and the treatment follows similar lines. Surgical correction should be carried out at the earliest opportunity and the principles are the same as those employed for the equino-varus deformity. The fool is approached in this instance from the lateral side. The tendo-calcaneus is elongated, the posterior capsule of the ankle joint divided, and the heel restored to its normal position, thus carrying the vertical talus into a more horizontal situation. In order to reduce the navicular onto the head of the talus, however, it is necessary to elongate peroneus longus, tertius and often the extensor tendons of the foot; to divide the tarsal capsular ligaments around the lateral side of the foot and to release peroneus brevis from its insertion. Relapse will be the inevitable sequel if this foot is not balanced, and it is the detached peroneus brevis that is used for this purpose. This tendon is passed through the interosseous membrane to the medial side and inserted into the tendon of tibialis posterior to augment its weakened action and maintain the medial arch of the foot. The whole procedure is identical to one described for the management of congenital vertical talus (Handelsman, 1968).

\section{Isolated Varus and Valgus Foot Deformities}

In a proportion of patients, the hind foot may be neutral with regard to equinus and calcaneus, but pulled into pure varus or valgus as the forefoot distorts following muscle imbalance. A pure varus deformity is unusual and can be rebalanced by a tibialis posterior transfer. A valgus foot, on the other hand, is commonly seen. When treated early, transfer of the peroneus brevis to the medial side of the foot or into the tibialis posterior tendon may be all that is required, but caution must be exercised when some function remains in the tibialis posterior or overaction may swing the foot from valgus to varus. In the older child, however, a tendon transfer may not be sufficient to correct the deformity, particularly when the heel valgus is severe. It is useful in these instances to do an extra-articular fusion of the subtalar joint so that the calcaneum may be held in a vertical position by the stable talus. Fusions in the feet of small children are not usually practicable or desirable, but in this instance, filling the sinus tarsi with a block of bone (Grice, 1952) will provide a fusion without interfering with articular surfaces. A simpler and more satisfactory method of achieving this fusion is to drive a resected length of the patient's fibular shaft (which soon regenerates) through a hole drilled in the neck of the talus and into the calcaneum, using a small incision on the dorsum of the foot. (Brown, 1968, Seymour and Evans, 1968). In the paralytic valgus foot, a subtalar arthrodesis alone will not maintain correction as the fusion will either melt away or distort with the passage of time, but as an augmentation procedure to a rebalancing operation it serves a very useful purpose. This principle holds true in other aspects of the management of deformity in myelomeningocoele.

\section{The Calcaneus Foot}

Paralysis of the triceps surae is not uncommon in these children and leads to a grotesque deformity. The calcaneum elongates and grows vertically downwards, termed aptly the "pistol grip heel" (Fig. 4a). Usually the long toe flexors are spared and their constant action in an attempt on the

Fig. 4: The typical "pistol grip heel" (a) is well illustrated in this 14-year old boy with a neglected calcaneo-valgus deformity. Tibialis anterior (b) and other tendons were inserted into the tendo-calcaneus which was then shortened, and bony correction achieved by excising a wedge of calcaneum and talus posteriorly. The staple provided fixation until sub-talar fusion was firm (c). The surgical correction is on the left foot, which previously resembled the deformity still present on the right side (d). Subsequently this foot was similarly treated with good result.
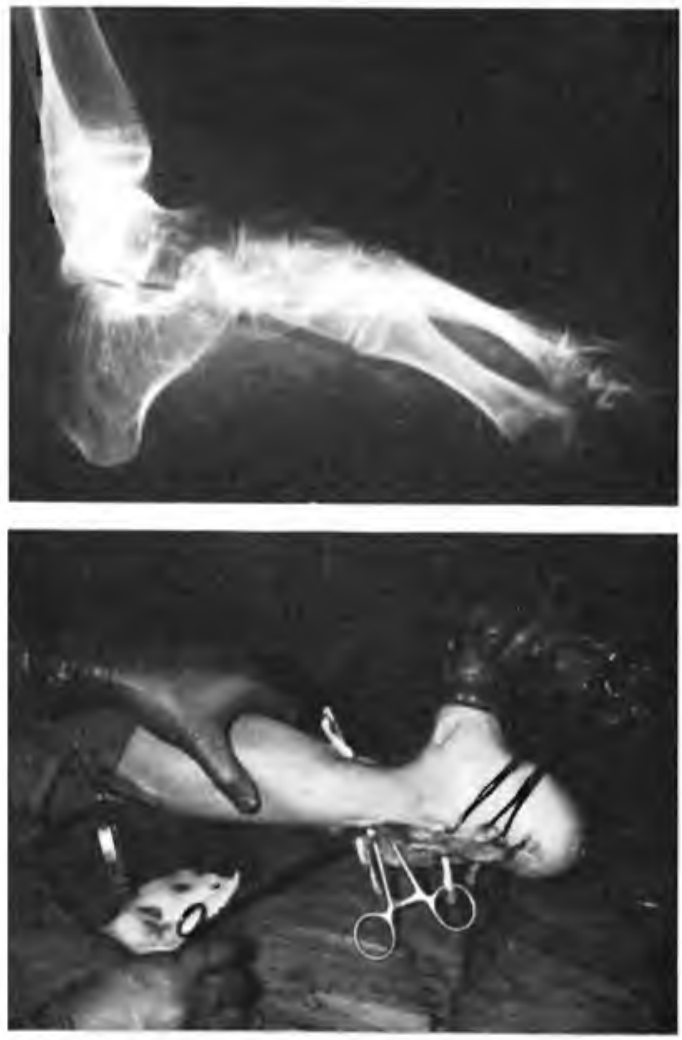

$4 c$

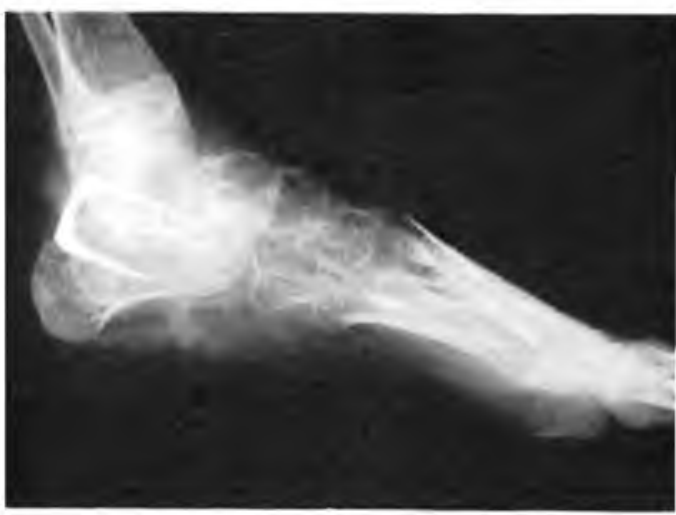

$4 d$

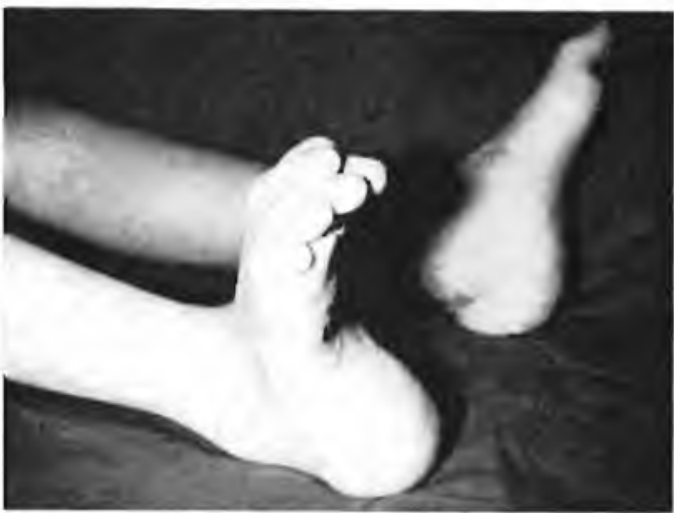


patient's part to bring the forefoot to the ground, combined with the slinging effect of the functioning peroneal tendons and Tibialis group, results in an equally unsightly cavus contracture that compounds the deformity (Fig. 4d). A combination of calcaneus and varus is unusual whereas the calcaneo-valgus foot is relatively common.

lf left untreated the deformity is not only unsightly but disabling because these children cannot bring the foot to the ground without bending the knees which soon develop fixed flexion contractures. The general principles of rebalancing apply to the calcaneus foot. In every instance the tibjalis anterior tendon and usually the peroneus tertius are rerouted into the tendo-calcaneus which is concurrently shortened (Fig. $4 b$ ). The remaining extensor digitorum longus provides adequate subsequent foot extension. When valgus is associated, one of the deforming tendons such as the peroneus brevis may also be transferred to the tendo-calcaneus or to the calcaneus itself, as may be tibialis posterior, should varus be present.

In the older child marked bony deformity becomes a feature, and tendon transfers alone will not suffice. A more normal appearance of the heel and much improved function is, however, quite easily achieved by excising a wedge of bone from the back of the calcaneum and talus to include the subtalar joint. The downward pointing heel may then be brought up into an anatomical position and subtalar fusion established by apposing the resected bony surfaces and fixing them with a staple (Fig. 4c).

Intrinsic Muscle Paralysis of the Foot

The main disability following paralysis of the interosseous and lumbrical muscles is the loss of flexor power of the metatarso-phalangeal joints. In attempting to compensate for this loss, the long flexors and extensors of the toes overact and ultimately the proximal phalanges sublux and later dislocate dorsally over the metatarsal heads and fixed flexion contractures of the inter-phalangeal joints develop. A cavus or cavo-varus deformity of the foot invariably follows.

The condition is amenable to rebalancing by detaching the short toe fexors from their insertions, re-routing the tendons alongside the proximal phalanges and inserting them into the common extensor tendons. In this new situation they replace the intrinsic muscles, and function as flexors of the metatarso-phalangeal joints and extensors of the inter-phalangeal joints. When performed sufficiently early, this flexor to extensor tendon transfer will prevent any fixed deformity. In the adolescent or older patient, however, fixed bony deformity has occurred and it may then be necessary to augment rebalancing procedures with a wedge excision and fusion in the mid-tarsal region to correct the cavus, or a valgus osteotomy of the calcaneum when the cavus is mild and the heel in varus.

\section{The Completely Flail Foot}

When the myelomeningocoele is high, paralysis in the foot is usually absolute and because there is no imbalance, deformities do not occur except as a result of gravity or the weight of bed clothes, which can produce equinus. These are readily prevented by physiotherapy and simple splintage.

Surgery in these patients is rarely indicated. Because it is unusual for them to have adequate control of the knees or hips, calipers remain necessary even if the feet are stabilised. It does occasionally happen, however, that a flail foot may be the only indication for an appliance and in these patients a triple arthrodesis of the foot may be worthwhile. In general terms foot stabilisation procedures in the myelomeningocoele child are unnecessary and often dangerous because, once the foot is in a fixed position, weightbearing may occur predominantly in a localised area and with deficient skin sensation, a pressure sore is difficult to avoid.

\section{The Knees}

Complete paralysis of the hamstring muscles allowing unopposed quadriceps function is common with the mid- lumbar spinal lesion. The knees are held in extension, often with some degree of recurvatum, and the range of flexion may be very limited soon after birth. With sitting, however, this usually improves and seldom requires treatment. On occasion it may be necessary to elongate the quadriceps tendon above the knee and very rarely a supracondylar osteotomy for severe recurvatum may be required.

Flexion contractures of the knee are much less common, and are seen in children who are able to walk but suffer from a severe calcaneus deformity. When the fixed extension of the feet is corrected, spontaneous correction of the knee deformity usually occurs and the treatment of these knees is the treatment of the deformity of the foot.

The wheel-chair cripple may develop a postural flexion contracture of the knee, but this usually responds to gentle stretching. Surgery is only occasionally necessary for the flexion contracture. When required, detachment of the shortened hamstrings and re-insertion into the back of the lower end of the femur and a posterior capsulotomy of the knee joint will usually secure correction. In the very rare case a corrective subtrochanteric osteotomy may be indicated. It is noteworthy however, that in fixed deformity a muscle may take on a function opposite to normal. For example, in a knee fixed in flexion, the ilio-tibial tract, which usually extends the knee, may become a flexor. Similarly in the recurved knee the sartorius and gracilis, normally flexors, become extensors in action and thus perpetuate the deformity. For this reason surgical correction must not be delayed when the deformities are fixed.

\section{The Hips}

By the age of 3 years, half the children suffering from myelomeningocoele will have dislocated hips. Approximately one third of this number would have been dislocated from birth. In all these children, the spinal lesion is in the mid-lumbar region, the hip flexors and adductors are spared but extensors and abductors are paralysed. This muscle imbalance produces, apart from a fiexion, adduction and external rotation contracture at the hip joint, a deformation of the neck of the femur because of an unopposed pull on the lesser trochanter. Experimental studies on a softened femur have established that a valgus and anteversion deformity of the femoral neck is the consequence of this form of muscle imbalance (Brookes and Wardle, 1962). The change in attitude of the femoral neck combined with an abductor weakness, produces an inevitable dislocation of the hip. Unlike the congenital type, when the dislocation is present from birth or soon thereafter, the acetabulum maintains a satisfactory shape and depth for several years (Fig. $5 a$ ). The subluxing hip on the other hand, produces a sloping acetabulum.

In the past, the hip dislocation of the myelomeningocoele child has usually been abandoned as an uncorrectable condition, or treated by varus and rotation femoral osteotomies, open reductions and shelf operations to deepen the acetabulum. In the face of muscle imbalance, however, the deformities recur and re-dislocation is inevitable. For the same reason, soft tissue releases such as the Soutter slide operation and adductor tenotomies produce poor results.

More recently Sharrard (1964) has produced a revolution in the management of these dislocated and contracted hips by combining a muscle balance operation with an open reduction and release procedure. The main deforming force in these young patients is the powerful iliopsoas muscle, which also provides a convenient replacement for the paralysed abductor group. In his operation, its tendon of insertion is removed from the lesser trochanter, routed through a hole made in the iliac blade, and re-inserted into the back of the greater trochanter. Once the iliopsoas is detached from the lesser trochanter, hip reduction becomes quite easy. Nevertheless the acetabulum should always be opened and cleared of fibrous tissue. In its new position the iliopsoas may well function as an abductor and extensor of the hip, but even where it does little beside provide a tenodesis effect, this is always sufficient to ensure continued 

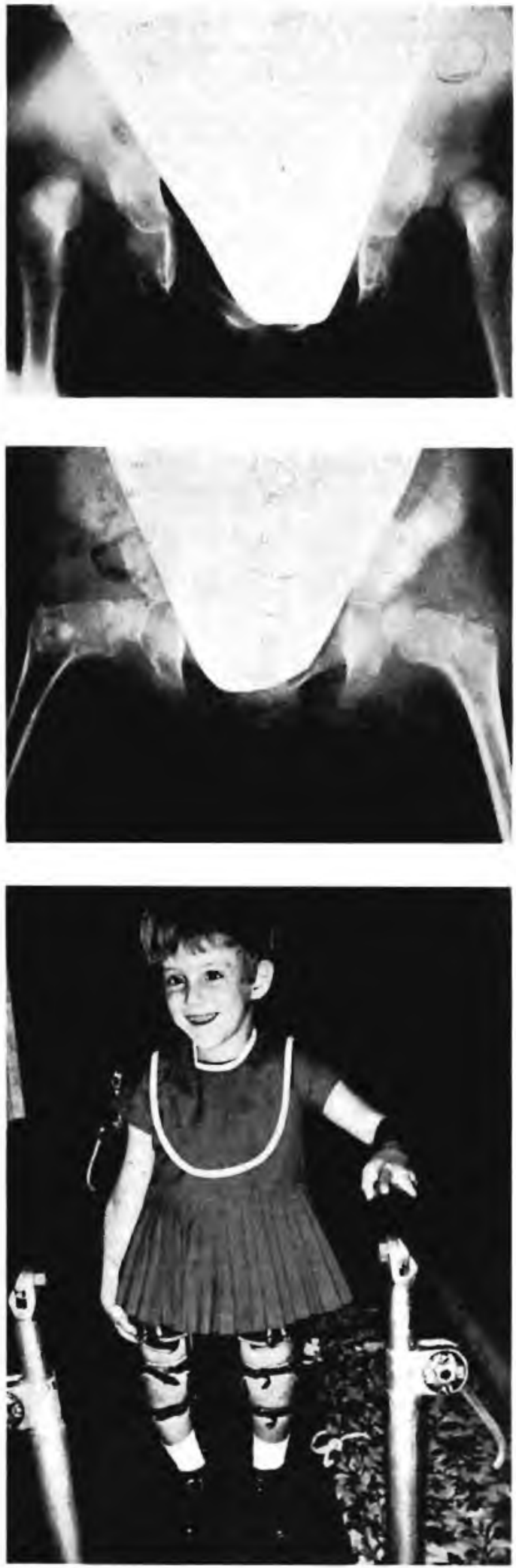

location of the hip joint and prevent recurrence of the flexion contracture. This operation is quite extensive but well worthwhile (Fig. $5 b$ and $c$ ).

Because the acetabulum usually remains well developed in the myelomeningocoele child, in most cases there is no harm in delaying the procedure until the child's general health is good and rehabilitation well established. Once the hip is reduced it is noteworthy that the valgus and anteversion deformity of the femoral neck gradually disappear even when patients are already in their early teens. In the older patient, the femoral neck may have to be corrected by a suitable osteotomy.

In the subluxing type of hip where acetabular shape is unsatisfactory, the iliopsoas transfer operation may conveniently be combined with a pelvic osteotomy (Salter, 1961) or shelf operation designed either to swing the acetabulum over the femoral head or to deepen it suitably.

After an iliopsoas transfer or combined operation, the patient is kept in a hip spica with the operated limb held abducted and internally rotated for $3 \underline{5}$ to 5 weeks and thereafter allowed free to rehabilitate. The results have been very satisfactory in reported series and confirmed by the 16 operations performed at the Spinal Defects Clinic of the Transvaal Memorial Hospital for Children.

\section{The Flail Lower Limb}

Where there is complete paralysis muscle imbalance cannot exist. The hips never dislocate and contractures are always secondary to postural or outside forces and respond to regular passive stretching. Physiotherapy to maintain full knee extension and plantigrade feet will allow the use of long-leg calipers and pelvic bands. Even when limited to a tripod gait, crutch walking in these patients is usually very satisfactory.

\section{SPONTANEOUS FRACTURES}

Approximately 25 per cent of our myelomeningocoele patients have sustained spontaneous fractures. Usually these follow periods of immobilisation in a hip spica after surgery. The fractures occur commonly through the metaphyseal region and are always painless, being diagnosed by abnormal mobility and soft tissue swelling.

It is well recognised that immobilisation in the supine position produces decalcification, nevertheless children with poliomyelitis undergoing similar operative procedures and immobilisation rarely develop spontaneous fractures. Furthermore, in some instances, fractures have occurred spontaneously in the mid-shaft of a bone without a period of immobilisation and with no trauma. These patients usually present with quite gross swelling of the affected leg or thigh, mild pyrexia, local heat and redness, but with no systemic toxic signs. A diagnosis of acute osteomyelitis is presumptive until the $\mathrm{X}$-ray reveals marked early callus in a very large sub-pereosteal haematoma.

The obvious bleeding under the periosteum is similar to scurvy, and it has been suggested (McKibben and Porter, 1967) that these patients have an abnormal Vitamin $C$ metabolism, resembling a chronic deficiency.

The fractures always heal rapidly and do not appear to present any long term problem but it would seem reasonable to provide additional Vitamin $C$ for these patients and to avoid undue immobilisation particularly in the supine position.

\section{REHABILITATION}

In the absence of severe mental deficiency, almost every myelomeningocoele patient can be taught to walk. Once deformities are corrected and the child is upright, the limiting factors are the degree of motivation in patient, parents and associated personnel.

Fig. 5: Bilateral dislocation of the hips in a 5-year old girl (a) were corrected by open reduction and iliopsoas transfers. The hole through which the transferred iliopsoas passed can be seen in the left iliac blade and the point of insertion of the transposed tendon is still visible in the right greater trochanter (b). An upright posture and assisted walking is now possible (c). 
Appliances are required to hold unstable joints but may never be used to attempt correction of a fixed deformity. When this exists, it is better to contour the appliance to the deformity than to endeavour to bend the limb to a rigidly designed splint. Calipers need to be carefully made to avoid pressure on insensitive skin and it is essential that patients and parents be taught to remove appliances and boots several times a day to inspect for signs of skin irritation. Boots with laces that extend to the toes are a valuable safety precaution, so that insensitive feet may be correctly situated under vision.

Below the knee, the use of inside and outside irons, T-straps, front and back stops all have the usual role to play in controlling various paralyses of the foot. A simple straight caliper is usually best for the unstable knee but as the child grows older it is advisable to fit a knee hinge to facilitate sitting at a school desk.

The importance of physiotherapy and occupational therapy cannot be over-emphasised. Centralisation of these services allows for group therapy that in itself provides an important boost for the patient. In this regard we are fortunate in having the facilities of the Hope School and Homes. Very good physical amenities exist, and staff enthusiasm and expertise are outstanding. The children progress steadily from the pool to parallel bars and ultimately to independent walking.

Total patient rehabilitation must not be neglected. Adequate schooling is essential and the provision of workshop and other facilities that can equip handicapped patients with a practical training will enable them to take their rightful place in society.

John Gallsworthy has observed "A niche of usefulness and a self-respect exists for every man however handicapped, but that niche must be found for him."

\section{SUMMARY}

The management of myelomeningocoele calls for an energetic and co-ordinated treatment regime that involves several disciplines. The orthopaedic surgeon must recognise and assess function. Insensitive skin precludes the use of manipulations, plaster casts and splints, and the treatment is surgical in the first instance. Division and elongation of tight structures will reverse otherwise progressive contrac- tures and joint dislocations, and selected muscle rebalancing by tendon transfers can prevent relapses. Joint arthrodesis may play a part but only in conjunction with rebalancing operations.

A subsequent unified programme of rehabilitation enables the majority of these patients to walk independently and many will lead useful lives.

\section{REFERENCES}

1. Brookes, M. and Wardle, E. M. (1962): Muscle action and the Shape of the Femur. Journal of Bone and Joint Surgery. 44B. 398.

2. Brown, A. (1968): A Simple Method of Fusion of the Subtalar Joint in Children. Journal of Bone and Joint Surgery. 50B. 364.

3. Grice, D. S. (1952): An Extra-Articular Arthrodesis of the Subastragular Joint for Correction of Paralytic Flat Feet in Children. Journal of Bone and Joint Surgery. 34A. 927.

4. Handelsman, J. E. (1968): Treatment of Congenital Vertical Talus. Journal of Bone and Joint Surgery. 50B. 439.

5. McKibben, B. and Porter, R. W. (1967): The incidence of Vitamin $\mathrm{C}$ Defficiency in Meningomyelocele. Develop. Med. Child Neurol. 9. 338.

6. Salter, R. B. (1961): Innominate Osteotomy in the Treatment of Congenital Dislocation and Subluxation of the Hip. Journal of Bone and Joint Surgery. 43B. 518.

7. Seymour, N. and Evans, D. K. (1968): A Modification of the Grice Sub-Talar Arthrodesis. Journal of Bone and Joint Surgery. 51B. 372.

8. Sharrard, W. J. W. (1964): Posterior lliopsoas Transplantation in the Treatment of Paralytic Dislocation of the Hip. Journal of Bone and Joint Surgery. 46B. 426.

9. Sharrard, W. J. W. (1964): The Segmental Innervation of the Lower Limb Muscles in Man. Annals of the Royal College of Surgeons of England. 35 (2). 106.

10. Sharrard, W. J. W. (1968): Spinal Osteotomy for Congenital Kyphosis in Myelomeningocoele. Journal of Bone and Joint Surgery. 50B. 466.

11. Solomon, L. and Handelsman, J. E. (1967): The Treatment of Club Foot. South African Journal of Surgery. 5 (1). 31. 